\title{
SUELO VIRTUAL Y DEUDA ECOLÓGICA. UN CÁLCULO PARA LA EXPANSIÓN DE LA SOJA EN ARGENTINA ${ }^{1}$
}

\author{
Federico Zuberman*
}

Resumen. Una de las características de la Economía Ecológica, es la de desarrollar indicadores del metabolismo social que permiten dar cuenta del grado de apropiación que hacen las sociedades de sus recursos naturales. En los últimos años vienen desarrollándose los indicadores de Suelo Virtual y Huella de Nutrientes los cuales intentan reflejar la presión que ejercen las sociedades sobre el suelo como recurso de base para la producción agropecuaria. Este trabajo intenta hacer una contribución en relación a la exportación de suelo virtual vinculada al cultivo de soja en Argentina desde su primera expansión como cultivo de exportación en 1970 hasta el año 2015. Por otro lado, sin aspirar a realizar un cálculo propio de la Economía Ambiental, intenta hacer una estimación monetaria de cuánto representarían esos nutrientes exportados en equivalentes de fertilizantes químicos. Como conclusión general se plantea a la huella de nutrientes y al suelo virtual como parte integrante del pasivo ambiental de Argentina.

Palabras Clave: Metabolismo Social; Huella de Nutrientes; Exportación de Nutrientes.

\footnotetext{
* Universidad Nacional de General Sarmiento (UNGS), Argentina.

Contacto: fzuberma@ungs.edu.ar

1 Una versión anterior fue presentada en las VIII jornadas de Economía Ecológica, Rosario, Argentina.
} 


\title{
VIRTUAL SOIL AND ECOLOGICAL DEBT. A CALCULATION FOR THE SOYBEAN EXPANSION IN ARGENTINA
}

\begin{abstract}
Ecological Economics is characterized, among other things, by developing indicators of social metabolism that allow accounting the level of appropriation of the natural resources by societies. In recent years, indicators as Virtual Soil and Nutrient Footprint have been developed with the aim of reflecting the pressure that societies makes on the soils as a basic resource for agricultural production. This paper tries to make a contribution in relation to calculate the export of virtual soil for the cultivation of soybean in Argentina since its first expansion as an export crop 1970 to 2015. Additionally, without performing a typical calculation of the Environmental Economics, it tries to make a monetary estimation of how much those nutrients exported represents in chemical fertilizer equivalents. In this way, as a general conclusion, we propose that virtual soil and nutrient footprint could be considered as a part of the ecological debt and environmental liability of our country
\end{abstract}

Keywords: Social metabolism; Nutrient footprin; Nutrient exportation

Original recibido el 12/01/2018

Aceptado para su publicación el 18/03/2019 


\section{Introducción}

La Economía Ecológica, entre otras características, se ocupa de desarrollar indicadores del metabolismo social que permitan dar cuenta del grado de presión que ejercen las sociedades sobre los recursos naturales e incluso de las desigualdades con las que dicha presión se expresa (Toledo, 2013; Martínez Alier, 2004). Existen diversos indicadores de esta índole que han resultado exitosos, tales como la Huella Ecológica (Wackernagel \& Rees, 1996), los Análisis de Flujos de Materiales (Fisher Kowalski y Hütler 1999), la Huella Hídrica y el Agua Virtual (Allan, 1993; Chapagain y Hoekstra, 2004), la Apropiación Humana de la Producción Primaria Neta-o HANPP por sus siglas en inglés- (Haberl, 1997), y otros. Los mismos se encuentran, en general, con un importante grado de estandarización y son utilizados o referenciados por diversas instituciones de carácter gubernamental y no gubernamental (EUROSTAT 2001; 2012; PNUMA 2013). En esa misma línea de indicadores, en los últimos años se ha avanzado con propuestas que reflejan la presión que ejercen las sociedades sobre el suelo, tanto como recurso productivo, como soporte y fuente permanente de nutrientes para los productos agrícolas y pecuarios. Dentro de este conjunto se destacan el Suelo Virtual (Pengue, 2010a; 2010b; 2010c; UNEP 2014; TEEB, 2018) y la Huella de Nutrientes (Pengue, 2014; Grönman et al. 2015).

Argentina, aún con sus diversos vaivenes en los modelos económicos adoptados históricamente, es un país fuertemente basado en la exportación de productos provenientes del agro. Esto se puede verificar, no solamente en términos del tradicional análisis sectorial de la economía y de los datos existentes de la balanza comercial monetaria. Desde la Economía Ecológica se presta especial atención al balance biofísico de la economía. En ese sentido, existen trabajos que analizan este tipo de balances biofísicos y reflejan que la mayor parte de las exportaciones en términos de materiales provienen de exportaciones de biomasa, seguidas de combustibles fósiles (Pérez Manrique et al. 2013; Zuberman y Fernández, 2016). Durante más de cien años Argentina se ha mantenido como uno de los principales proveedores de productos agrícolas a nivel global, y particularmente, en las últimas décadas ha triplicado su producción fundamentalmente a raíz de la expansión de la soja (Zuberman, 2014). La introducción de la soja transgénica RR (Roundup Ready) en la campaña 1996/97 y su paquete tecnológico de siembra directa y labranza química mediante glifosato marcaron un punto de inflexión en la producción agrícola argentina. En menos de dos décadas se pasó de 6 millones de hectáreas sembradas -es decir exactamente un cuarto de la superficie agrícola sembrada en el país y entre un $15 \%$ y un $20 \%$ de las toneladas producidas- a más de 20 millones de hectáreas sembradas, implicando actualmente casi la mitad de su producción agrícola y más de un $50 \%$ de la superficie cultivada (Ver Gráfico 1). Dicha expansión, como también lo refleja el Gráfico 1, se ha dado no solo a costa del avance de la frontera agrícola sino también en reemplazo de otros cultivos. 
Gráfico 1. Superficie sembrada con soja y superficie sembrada con el resto de los cultivos (sin incluir soja) desde 1969/70 a 2014/2015. En hectáreas.

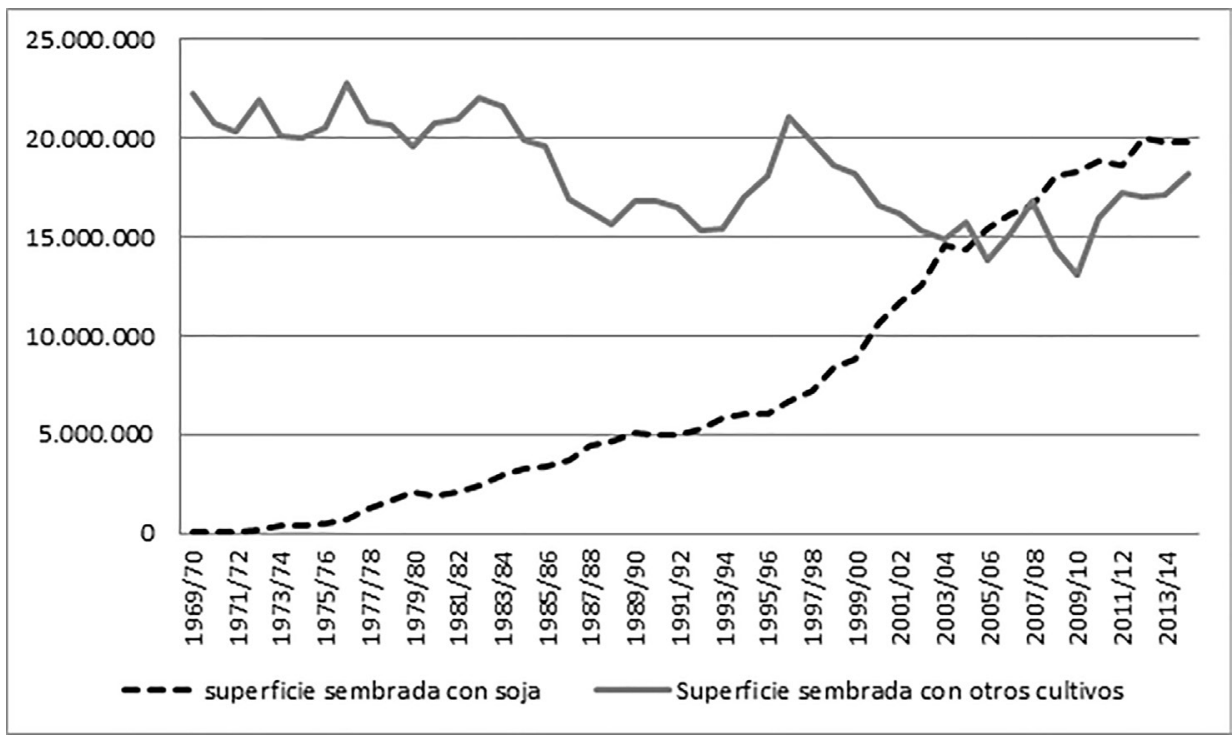

Fuente: elaboración propia en base a datos del Servicio de Estimaciones Agrícolas -Datos de la Dirección de Información Agrícola y Forestal del Ministerio de Agroindustria de la República Argentina.

En ese sentido resulta relevante hacer distintos tipos de análisis que den cuenta del impacto que pueda generar la expansión de la agricultura de exportación sobre los suelos. En este caso, más aún pues al tratarse de un solo cultivo (o peor aún, en muchos casos una falsa rotación de trigo-soja continua) estamos en presencia de un proceso de extracción selectiva de nutrientes. Es importante además que estos análisis puedan evaluar la problemática de estos niveles de extracción no solo a nivel parcela o establecimiento sino a nivel macro, a escala regional y en una escala temporal de largo alcance. El presente trabajo se propone contribuir con este tipo de análisis haciendo una aproximación al cálculo de Suelo Virtual concentrándose en el avance del cultivo de soja en Argentina desde la campaña 1969/70 hasta la campaña 2014/2015.

Dada la dependencia de los países latinoamericanos para sostener el saldo positivo de sus balanzas comerciales con las exportaciones de materias primas basadas en un uso intensivo de recursos naturales, la pérdida de fertilidad de los suelos puede considerarse como parte de la Deuda Ecológica que pesa sobre sus economías y que al día de hoy se sigue incrementando (Pengue, 2005). En ese sentido el cálculo de Suelo Virtual realizado se complementa con un cálculo monetario sobre la implicancia de esta exportación de nutrientes. Esto no intenta reducir el análisis a un cálculo crematístico, propio de la Economía Ambiental, sino acompañar al indicador metabólico social mencionado con cifras que sirvan como patrón para la discusión y que colaboren a tomar noción de nivel de Deuda Ecológica generada. Para ello 
se calculó qué cantidades de fertilizantes resultarian equivalentes a la exportación acumulada de seis macronutrientes y siete micronutrientes, y luego se calculó el costo monetario que implicarían dichas cantidades de fertilizantes.

\section{Metodología aplicada}

Suelo Virtual y Huella de Nutrientes son dos indicadores novedosos y ciertamente emparentados que se están comenzando a utilizar para poder dar cuenta a grandes escalas, tanto espacial como temporal, de la presión sobre el recurso suelo que realiza la actividad agropecuaria, en especial aquella ligada a exportación a través de mercados globales (UNEP, 2014; TEEB 2018; Díaz de Astarloa y Pengue, 2018). En ese mismo sentido, al ser conceptos recientes aún no tienen un método concluido y estandarizado de cálculo. En vistas de lograr tal proceso de estandarización también es que se ofrecen estos resultados.

Según Pengue (2010c, 2014, 2017) el Suelo Virtual representa la cantidad de nutrientes que son extraídos del suelo, y se exportan contenidos en los productos agrícolas o pecuarios exportados. De este modo se intenta reflejar la depleción (disminución o agotamiento) de los nutrientes en los espacios de producción, poniendo énfasis en los flujos internacionales de estos productos y vinculando los costos ambientales de los territorios donde éstos se producen con sus lugares de consumo.

Esta definición de Suelo Virtual remite a la componente Extracción Total incluida en la fórmula de la Huella de Nutrientes, indicador que según Pengue (2014) puede resumirse en la siguiente fórmula:

$$
\text { Huella de Nutrientes }=S+\Sigma F i z+F B N-\Sigma C i j P j k
$$

Siendo: $S=$ Stock de nutrientes; $F=$ fertilizante; $i=$ nutrientes; $z=$ cultivo; $F B N=$ Fijación Biológica (i.e. el caso del Nitrógeno); $C=$ coeficiente de extracción; $P=$ producción; $i=$ nutrientes; $j=$ grano; $k=$ Departamentos/Partidos; donde

$$
\text { Extracción Total }=\Sigma \text { CijPjk y Reposición Total }=F B N-\Sigma C i j P j k
$$

Como se observa, la Huella de Nutrientes presentada aquí es casi equiparable al frecuentemente utilizado Balance Total Simplificado, el cual cuantifica en un análisis de flujo de caja cerrada, al balance entre el flujo de ingresos (vía fertilizantes, fijación simbiótica y aportes de biomasa residual) y salidas (vía cosechas de granos, frutos, carne, leche o fibras), de los nutrientes de un determinado espacio productivo (Pengue, 2014).

Aquellos productos que salen del sistema local, sea a través de granos, frutos, carne, leche, madera, etc. se convierten en pérdidas físicas nutricionales del mismo, en la medida que no se repongan. Esto es lo que intenta contemplar el Suelo Virtual, que como se dijo, es equiparable a la componente Extracción Total de la Huella de Nutrientes

En este trabajo se realizó un cálculo de Suelo Virtual analizando lo concerniente al avance del cultivo de soja en la Argentina durante los últimos 45 años por ser un caso paradigmático de rápida expansión territorial y por ser el cultivo de mayor volumen producido en el país y en la región. En efecto, algunos trabajos le atribuyen 
a la soja entre un $45 \%$ (Flores y Sarandón, 2003) y un 54\% (Díaz de Astarloa y Pengue, 2016; 2018) de la extracción total del Nitrógeno (N), Fósforo (P) y Potasio (K) hecha en el país.

La estimación realizada calcula tanto la exportación de seis macronutrientes $(\mathrm{N}, \mathrm{P}, \mathrm{K}$, $\mathrm{S}, \mathrm{Ca}, \mathrm{Mg}$ ) como de siete micronutrientes (B, Cl, $\mathrm{Cu}, \mathrm{Fe}, \mathrm{Mn}, \mathrm{Mo}, \mathrm{Zn}$ ).

Para el cálculo de la ecuación propuesta los valores de $C$ (coeficiente de extracción) fueron los utilizados por Pengue (2014) en tanto que los de $P$ (producción) fueron tomados del Servicio de Estimaciones Agrícolas - Datos de la Dirección de Información Agrícola y Forestal del Ministerio de Agroindustria de la República Argentina.

Por último, se realizó un cálculo del costo actual de tales cantidades de nutrientes equivalentes en fertilizantes químicos. Para ello, se tomó el peso equivalente de cada nutriente en la formulación comercial de los siguientes fertilizantes químicos: urea granulada, superfosfato triple, sulfato de amonio, cloruro de potasio y dolomita.

\section{Resultados y discusión}

\subsection{Cálculo de Suelo Virtual.}

En base a las estimaciones realizadas la Argentina entre 1970 y 2015 ha exportado a través de la soja: 25,5 millones de t de nitrógeno, 5,7 millones de t de fósforo, 16,6 millones de $t$ de potasio, 2,6 millones de t de calcio, 2,3 millones de t de magnesio y 4 millones de t de azufre. Para el caso de los micronutrientes contabilizan 21.284 t de Boro, 201.776 t de Cloro, 21.284 t de Cobre, 255.413 t de Hierro, 127.706 t de Manganeso, $4.257 \mathrm{t}$ de Molibdeno y $51.083 \mathrm{t}$ de Zinc. Tal como lo muestran los Gráficos 2 y 3, el principal salto en la exportación de nutrientes se da a partir de la liberación de la soja transgénica en la campaña de 1997 y la tendencia no parece revertirse. Más allá de algún excepcional año de baja productividad, su expansión continuó a ritmo sostenido en las siguientes dos décadas. No está demás señalar que tal recrudecimiento en la exportación de nutrientes no se debe tanto a la condición de la soja RR de ser un cultivo transgénico como al marco socioeconómico favorable que ha hecho posible su rápida expansión (Teubal, 2006). En ese sentido, ni los nuevos paquetes tecnológico-productivos ni el modelo de agronegocios con el que se expande este tipo de agricultura, se pueden analizar separadamente de las externalidades que generan. 
Gráfico 2. Extracción, estimada en toneladas, de seis macronutrientes para las campañas 1969/1970 a 2014/2015 en el cultivo de soja en Argentina.

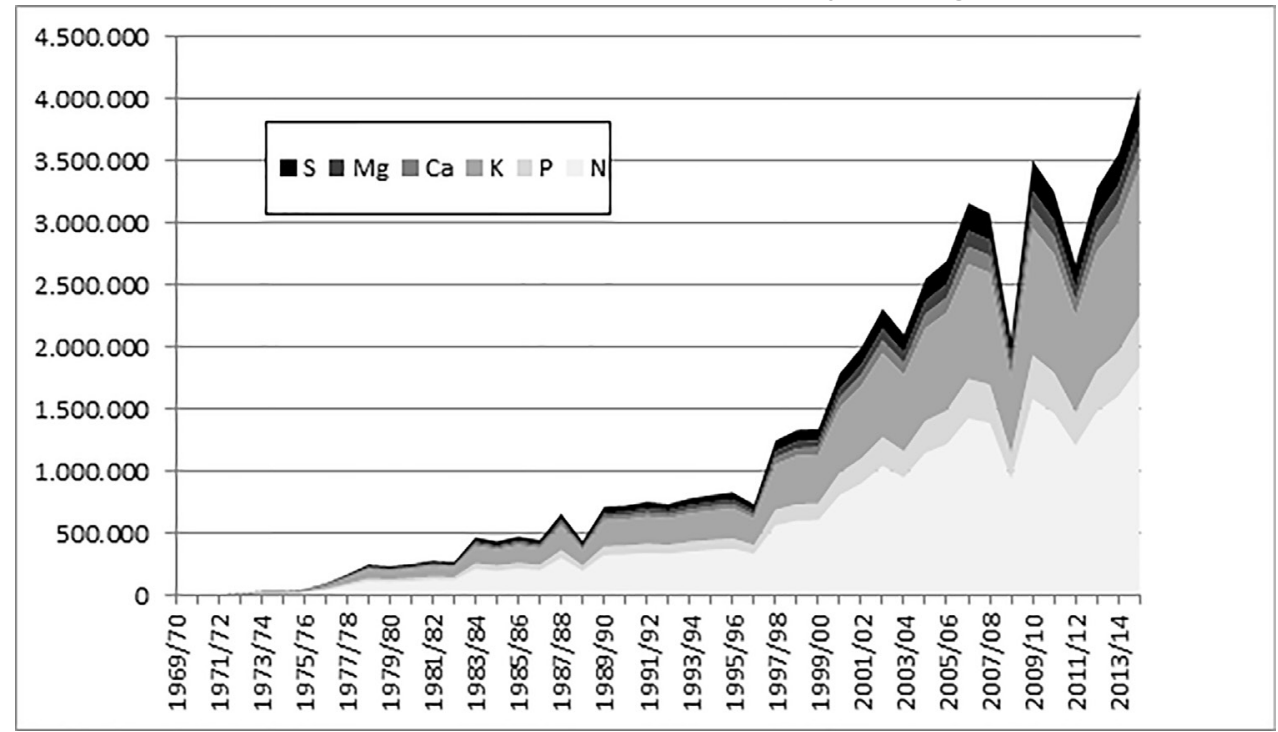

Fuente: elaboración propia en base a datos del Servicio de Estimaciones Agrícolas - Datos de la Dirección de Información Agrícola y Forestal del Ministerio de Agroindustria de la República Argentina y coeficientes de extracción utilizados por Pengue (2014).

Gráfico 3. Extracción, estimada en toneladas, de algunos micronutrientes para las campañas 1969/1970 a 2014/2015 en el cultivo de soja en Argentina.

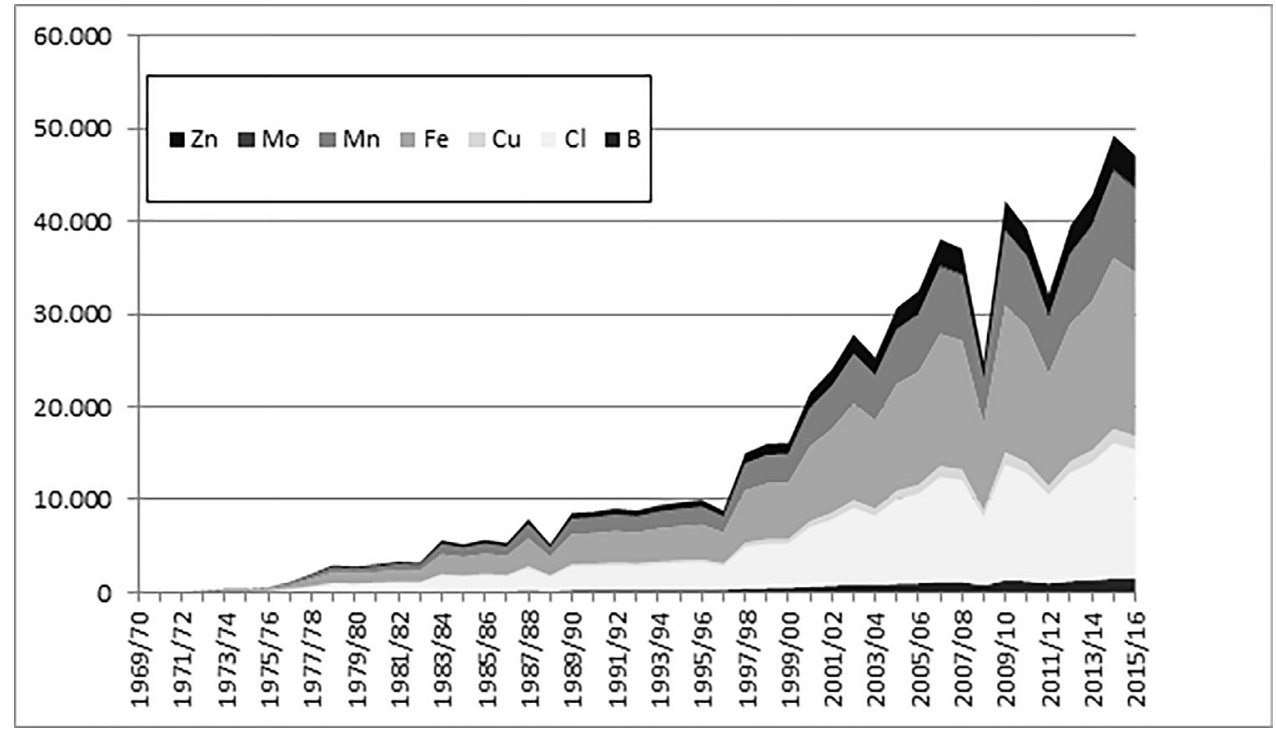

Fuente: elaboración propia en base a datos del Servicio de Estimaciones Agrícolas - Datos de la Dirección de Información Agrícola y Forestal del Ministerio de Agroindustria de la República Argentina y coeficientes de extracción utilizados por Pengue (2014).

SaberEs. Vol. 11, Núm.1 (2019). 81-95 Sección Artículos 
La pérdida de nutrientes durante el período considerado prácticamente duplica las cifras que se estimaron hasta el año 1999 (Flores y Sarandón, 2003). Es decir que, aun habiendo advertido esta problemática hace más de 15 años y evidenciada en distintas publicaciones científicas, el problema ha continuado agravándose.

Como se mencionó anteriormente, estas cifras apenas representan la porción de nutrientes que se exporta a través de los granos de soja cosechados. Es decir que no contemplan ni las reposiciones naturales (sobre todo la fijación biológica, tratándose de una leguminosa) ni artificiales (fertilizaciones), ni otros problemas tanto más graves que involucran pérdida de nutrientes como la erosión o la pérdida de materia orgánica. Pero incluso con estas limitantes es interesante notar que estos resultados se condicen con los niveles de déficit nutricional en suelos argentinos advertidos por Viglizzo y Jobbagy (2010) y Cruzate y Casas (2009).

La mayor parte de la reposición de nutrientes en nuestro país se hace vía fertilización química. Sin embargo de acuerdo a las estimaciones disponibles solo se ha repuesto entre un $15 \%$ y un $30 \%$ del $\mathrm{N}$ y un $40 \%$ del $\mathrm{P}$ (Cruzate y Casas, 2012; Díaz de Astarloa y Pengue, 2018). Pero aun reponiendo una cantidad de nutrientes idéntica a la exportada, el problema alcanza varias aristas que no llegan a ser tratadas adecuadamente. En primer lugar porque gran parte de estas fertilizaciones se pierde (Rimski-Korsakov et al. 2008). Para el caso del nitrógeno, la fracción que no es tomada por los cultivos puede ser considerable. Según Collins et al. (2007) la eficiencia en el uso del $\mathrm{N}$ por los cultivos varía entre $33 \%$ y $50 \%$, a causa de la fluctuación temporal y espacial de los nitratos en el perfil del suelo, entre otros factores. Otros trabajos hablan de hasta de un $60 \%$ de pérdida (Macdonald et al. 1997; Aparicio et al. 2015). Ese nitrógeno que no es tomado por los cultivos puede tener como destinos la atmósfera, por volatilización y desnitrificación o las napas subterráneas, por lixiviación. Si bien el primero, en su forma de óxido nitroso (N2O) es cada vez más problemático en términos de gases de efecto invernadero (Crutzen et al. 2007), es de particular importancia el N perdido por lixiviación. Esta fuente de contaminación tiende a elevar los niveles de nitratos y nitritos en napas subterráneas (Rimski-Korsakov et al. 2004; Civiera y Rodríguez, 2011). Pero además de ser un problema ambiental de alcance local, por la consecuente eutrofización de distintos cuerpos de agua, forma parte de un problema ambiental a escala global, conocido como efecto cascada del Nitrógeno, que según Rockstrom et al. (2009) ya ha sobrepasado el límite de sostenibilidad planetaria. Por otra parte, la acidificación que genera el continuo proceso de utilización de agroquímicos también puede inmovilizar otros macronutrientes, como el fósforo, lo cual podría terminar en un círculo vicioso generando una mayor dependencia de nuevos agregados de fertilizantes.

En la discusión sobre el uso de fertilizantes químicos, resulta interesante señalar que aun habiéndose incrementado notoriamente el uso de los fertilizantes, sobre todo desde la década del noventa (García y González San Juan, 2010), al haber mayor extracción por la expansión e intensificación de los cultivos, el déficit de nutrientes se hace cada vez mayor, particularmente en nutrientes como el Nitrógeno. Del mismo modo, si bien se puede inferir que la expansión de la soja permite una mayor fijación 
biológica de nitrógeno, el nitrógeno exportado resulta mayor, incrementando de igual manera el déficit en el suelo (Díaz de Astarloa y Pengue, 2018)

Por otro lado, también resulta interesante resaltar que el carbono (C) no está incluido en este cálculo porque no forma parte del stock de nutrientes que son incorporados desde el suelo a la planta. Sin embargo pueden resultar de particular interés en la discusión sobre el metabolismo social del recurso suelo, no solo por el hecho de que el flujo internacional de biomasa es naturalmente un flujo de carbono sino porque el suelo es uno de los reservorios de carbono más importantes, conteniendo aún más carbono que la atmósfera y la vegetación terrestre en su conjunto (Gruijter et al, 2016). En ese sentido la degradación de los suelos y la consecuente mineralización de la materia orgánica contenida en los mismos implican una notable alteración de la dinámica de este ciclo biogeoquímico, liberando grandes cantidades de dióxido de carbono y otros gases de efecto invernadero a la atmósfera (FAO, 2017).

Los términos Huella de Nutrientes y Suelo Virtual pueden ser entendidos como cierta analogía a algunos indicadores ya lo suficientemente estandarizados como son la Huella Hídrica y el Agua Virtual. Sin embargo, desde su conceptualización y construcción, tienen importantes diferencias que vale la pena señalar para evitar confusiones en el uso de los mismos y en su referenciación. En primer lugar, lógicamente, el recurso agua tiene una dinámica muy distinta a la del suelo. Pero más allá de eso, la diferencia en el indicador está en que la Huella Hídrica y el Agua Virtual (Hoekstra 2003; Chapagain y Hoekstra, 2004; Pengue, 2006) no reducen su cuantificación al agua que sale del sistema contenida en los productos exportados sino que contabiliza el agua utilizada tanto directamente como indirectamente en todo el proceso de producción. El Suelo Virtual, por el contrario, es apenas la porción de nutrientes que se encuentra contenido en esos productos exportados con lo cual no está cuantificando la alteración en el balance nutricional que pueda sufrir el suelo por encima de eso. Todo tipo de deterioro en la caja de nutrientes del suelo que se deba a la mineralización acentuada de la materia orgánica, por un uso cada vez más intensivo del suelo, la nitrificación, la baja en la disponibilidad de Fósforo por cambios en el pH y su adsorción a los coloides, o el deterioro de los macroagregrados, no se estaría cuantificando dentro de este indicador. A su vez la erosión (hídrica o eólica) también representa pérdidas de suelo que no están siendo cuantificadas pero que bien podrían empezar a correlacionarse. Queda claro que en la medida en que las urgencias económicas de corto plazo conduzcan a intensificar el uso del suelo, disminuyendo las necesarias rotaciones agrícolas-ganaderas y la alternancia de cultivos o reemplazando ecosistemas de alta fragilidad por planteos agrícolas continuos, el proceso se agravaría notablemente.

\subsection{Estimación monetaria del costo de los nutrientes exportados}

La Economía Ecológica cuestiona la idea de reducir el análisis metabólico social a un indicador monocriterial como es el dinero. Tal es la metodología utilizada por la Economía Ambiental y lamentablemente viene siendo la más difundida entre los intentos de vincular análisis ambientales con los económicos. En ese sentido, este trabajo no pretende caer en el mismo reduccionismo. Luego de haber analizado la propia dinámica de exportación de nutrientes del suelo y comprender los lazos que la 
vinculan a una dinámica económica y social, se hizo un cálculo de ese tipo, no con el fin de reducir el análisis a un valor monetario sino agregarlo como posible patrón de comparación con otros elementos numéricos de la economía Argentina.

En ese sentido, se ha hecho un cálculo del costo monetario que implica apenas la recuperación de los macronutrientes perdidos en todo el período considerado (1969-2015) (sin contar costos de incorporación al suelo y suponiendo que todos esos nutrientes resultan efectivamente incorporados al suelo) mediante el uso de fertilizantes químicos. Para el caso de los micronutrientes, su reposición vía fertilizantes suele reducirse a frutales, hortícolas y a la fruticultura. En cultivos extensivos, como es el caso de la soja, en general se suele considerar que la carga de micronutrientes presente en los fertilizantes químicos utilizados alcanza a compensar su extracción. Sin embargo, algunos trabajos ya han encontrado una importante cantidad de respuestas positivas a la fertilización con micronutrientes en soja (Ferraris y Couretot, 2011). Por otra parte, su disponibilidad está estrechamente relacionada a los cambios de $\mathrm{pH}$ del suelo.

Haciendo el cálculo en base al peso equivalente de cada nutriente en cada una de las formulas moleculares de los tipos de fertilizantes más utilizados, el número estaría cerca de los 50.000 millones de dólares (16.797.507.560 de urea granulada, 13.627.204.800 de SPT, 10.971.264.666,67 de Cloruro de Potasio, 5.734.868.611,11 de Sulfato de Amonio, y 2.535.270.000 de Dolomita). Los precios de referencia de los fertilizantes fueron tomados para el mes de abril de 2017. Se aclara que no hay doble contabilización ni para el caso del $\mathrm{N}$ (entre el $\mathrm{N}$ aportado por la urea granulada y el presente en el Sulfato de Amonio alcanzan las 25.541.289 toneladas de nitrógeno necesarias) ni para el caso del S (entre el azufre aportado vía el sulfato de amonio y el azufre presente en la dolomita suman las 3.992.955 toneladas mencionadas en el apartado anterior).

Para poder referenciar esta cifra en términos actuales, el monto acumulado equivale a alrededor de un 10\% del PBI del año 2016; algo bastante cercano a lo que representa el PBI agropecuario actualmente o bien, para dar otro equivalente, casi el triple del total de las exportaciones del complejo sojero que en 2016 alcanzó los 17.000 millones de dólares.

Como se manifestó anteriormente, este cálculo es una supersimplificación que no tiene en cuenta la problemática que implica la compleja relación de alguno de estos nutrientes en particular (por ejemplo el P, que por su condición de inmóvil incrementa los riesgos de agotamiento, o el $\mathrm{N}$ que por su condición de móvil podría contaminar napas subterráneas) ni los costos ambientales de usar este tipo de agroquímicos. Por eso, debe quedar claro que sería erróneo concluir en que el pasivo ambiental debido a la exportación de nutrientes por la exportación de soja desde la década del setenta se reduzca a los 50.000 millones de dólares mencionados.

\subsection{Deuda Ecológica}

Hace varios años se viene desarrollando la idea de una Deuda Ecológica que el norte le debe al sur y discutiendo sobre su posible cuantificación (Pengue, 2002; Martínez Alier, 2008). Su origen en nuestro continente bien puede remontarse a una deuda colonial, donde el saqueo de metales preciosos, el agotamiento de suelos 
arrasados por monocultivos y el desmonte de bosques y selvas enteras, enriqueció las arcas de las metrópolis europeas y financió buena parte de su desarrollo. Pero la misma se extiende hasta nuestros días a través de un comercio ecológicamente desigual, donde las economías de la periferia se ven obligadas a exportar cada vez mayores cantidades sus recursos naturales para acceder a los bienes proveídos, casi exclusivamente, por los países centrales. Se trata, en definitiva, de un correlato ecológico de la teoría del deterioro de los términos del intercambio planteada por Prebisch (1979; 1986).

La idea de Deuda Ecológica es hija de las discusiones sobre deuda externa (Schatan, 1998). Aquellas sumas siderales de dinero que fueron contraídas por la mayor parte de los países de la periferia -muchos de ellos en períodos de ilegítimos gobiernos dictatoriales avalados o promovidos por Washington- con la ilusión de generar la industrialización, modernización y el desarrollo de sus economías, acuciaron durante varias décadas los presupuestos de estos países. Impagables intereses que crecían exponencialmente fueron pagados no solo a costa de una presión económica sobre las sociedades sino también sobre sus recursos naturales. En ese sentido, la Deuda Ecológica ha sido definida por Donoso (citada en Pengue, 2002) como aquella que ha venido siendo acumulada por el Norte, especialmente por los países más industrializados hacia las naciones de la periferia a través de la expoliación de los recursos naturales, por su venta subvaluada, la contaminación ambiental, la utilización gratuita de sus recursos genéticos o la libre ocupación de su espacio ambiental para el depósito delos gases de efecto invernadero u otros residuos acumulados y eliminados por los países industrializados. De esta forma, la exportación de nutrientes, bien puede ser parte constitutiva de la misma, sobre todo en un país de base agroexportadora como Argentina.

Por otra parte en un contexto como el actual, luego de un período de inusitado crecimiento económico pero con un discutible grado de reprimarización y a las puertas de un nuevo ciclo de endeudamiento externo, cobra nuevamente dimensión la discusión sobre deuda ecológica y su necesidad de cuantificar ciertos pasivos ambientales.

\section{Conclusiones}

El Suelo Virtual puede ser un interesante indicador del metabolismo social que ayude a determinar el grado de presión que ejerce la sociedad sobre el suelo como recurso fundamental para la producción de alimentos. En la medida que los países del cono sur incrementan la presión sobre este recurso, en el afán de incrementar sus exportaciones para, en la mayoría de los casos, sostener la balanza comercial, están comprometiendo sus cosechas futuras. En ese sentido la exportación de nutrientes no solo es una deuda con las generaciones futuras, sino que es parte de la deuda ecológica que, a nivel global, continúan contrayendo los países del norte a costa de la importación infravalorada de recursos naturales de los países del sur. Aportes como este sirven para comenzar a contar con estimaciones que cuantifiquen los déficits nutricionales del suelo así como para referenciar la subestimación con la que se gestiona el soporte natural que sostiene gran parte de la base de nuestra economía monetaria. 


\section{Referencias bibliográficas}

Allan, J. A. (1993). Fortunately there are substitutes for water otherwise our hydropolitical futures would be impossible. Priorities for water resources allocation and management, 13(4), 26.

Aparicio, V., Costa, J. L., Sainz Rozas, H., Giménez, D., y García, F. (2015). Comparing nitrate- $\mathrm{N}$ losses through leaching by field measurements and nitrogen balance estimations. Communications in soil science and plant analysis, 46(10), 1229-1243.

Civeira, G. y Rodríguez, M. (2011). Nitrógeno residual y lixiviado del fertilizante en el sistema suelo-planta-zeolitas. Ciencia del Suelo, 29(2), 285-294.

Chapagain, A. K. y Hoekstra, A. Y. (2004). Waterfootprints of nations, value of water research. Report Series No. 16. Delft, Holanda: UNESCO-IHE.

Collins, H. P., Delgado, J. A., Alva, A. K. y Follett, R. F. (2007). Use of Nitrogen-15 Isotopic Techniques to Estimate Nitrogen Cycling from a Mustard Cover Crop to Potatoes. Agronomy journal, 99.

Cruzate, G. y Casas, R. (2009). Extracción de nutrientes en la agricultura argentina. Argentina. Informaciones agronómicas del Cono Sur, 44, 21-26.

Cruzate, G. y Casas, R. (2012). Extracción y balance de nutrientes en los suelos agrícolas de la Argentina. Informaciones agronómicas de Hispanoamérica, 6, 7-14.

Crutzen, P. J., Mosier, A. R., Smith, K. A. y Winiwarter, W. (2007). N2O release from agro-biofuel production negates global warming reduction by replacing fossil fuels. Atmospheric Chemistry and Physics Discussions, European Geosciences Union, 11191-11205.

Díaz de Asterloa, D. y Pengue, W. (2016). Soils Health and Agriculture Sustainability: Nutrients Metabolism in Argentina. One Health Eco Health Conference. Melbourne, Australia.

Díaz de Asterloa, D. y Pengue, W. (2018). Nutrients Metabolism of Agricultural Production in Argentina: NPK Input and Output Flows from 1961 to 2015. Ecological Economics, 147, 74-83.

EUROSTAT. (2001). Economy-wide Material Flow Accounts and derived indicators. A methodological guide. Luxemburgo: Office for Official Publications of the European Communities.

EUROSTAT. (2012). Economy-wide Material Flow Accounts (EW-MFA). Compilation Guide. Luxemburgo: Office for Official Publications of the European Communities. 
FAO. (2017). Carbono Orgánico del Suelo: el potencial oculto. Roma, Italia: Organización de las Naciones Unidas para la Alimentación y Agricultura.

Ferraris, G. y Couretot, L. (2011). Fertilización con micronutrientes en soja. Experiencias en la región centro - norte de Buenos Aires y sur de Santa Fe. Mercosoja 2011, "Un grano, un Universo". Rosario, Argentina.

Fisher Kowalski, M. y Hütler, W. (1999). Society's Metabolism. The intellectual History of Materials Flow Analysis, Part II, 1970 - 1998. Journal of Industrial Ecology, 2(4), 107-136.

Flores, C. C. y Sarandón, S. (2003). ¿Racionalidad económica versus sustentabilidad ecológica? El ejemplo del costo oculto de la pérdida de fertilidad del suelo durante el proceso de agriculturización en la Región Pampeana Argentina, Argentina. Revista de la Facultad de Agronomía, 105(1), 52-67.

García F. y González San Juan, M. F. (2010). Balances de nutrientes en Argentina ¿Cómo estamos? ¿Cómo mejoramos? Informaciones Agronómicas del Cono Sur, 48, 1-5.

Grönman, K., Ypyä, J., Virtanen, Y., Kurppa, S., Soukka, R., Seuri, P., Finér, A. y Linnanen, L. (2015). Nutrient footprint as a tool to evaluate the nutrient balance of a food chain. Journal of Cleaner Production, 112(4), 2429-244.

Gruijter, J. J., Mc Bratney, A. B., Minansy, B., Wheeler, I., Malone, B. P. y Stockman, U. (2016). Farm-Scale soil carbon auditing. Geoderma, 120-130.

Haberl, H. (1997). Human Appropriation of Net Primary Production as An Environmental Indicator: Implications for Sustainable Development. Ambio, 26(3).

Hoekstra A. (Ed.) (2003). Virtual water trade. Proceedings of the International Expert Meeting on Virtual Water Trade. Value of Water Research Report Series, 12. Delft, Holanda.

Macdonald, A. J., Poultron, P. R., Powlson, D. S. y Jenkinson, D. S. (1997). Effects of season, soil type and cropping on recoveries residues and losses of $15 \mathrm{~N}$-labelled fertilizer applied to arable crops in spring. The Journal of Agricultural Science, 129, 125-154.

Martínez Alier, J. (2004). Los conflictos ecológico-distributivos y los indicadores de sustentabilidad. Revista Iberoamericana de Economía Ecológica, 1, 21-30.

Martínez Alier, J. (2008). Cuantificación de la deuda ecológica. En ALC, Sur: Soberanía y Dignidad. No Somos Deudores, Somos Acreedores. Memorias del encuentro Alianza de Pueblos del Sur Acreedores de Deudas Históricas, Sociales y Ecológicas. Quito, Ecuador: SPEDCA - ALC.

Pengue, W. (2002). La pampa sin el ombú: comercio ecológicamente desigual y deuda ecológica. Realidad Económica, 190. 
Pengue, W. (2005). Agricultura Industrial y Transnacionalización en América Latina ¿La transgénesis de un continente? PNUMA, UACM y GEPAMA.

Pengue, W. (2006). "Agua virtual", agronegocio sojero y cuestiones económico ambientales futuras. Revista Fronteras, 5, 14-25.

Pengue, W. (2010a). Suelo virtual y comercio internacional. Realidad Económica, 250.

Pengue, W. (2010b). Agricultura mundial, suelo virtual y agrocombustibles. Cuaderno Interdisciplinar de desarrollo sostenible, 4.

Pengue, W. (2010c). Suelo virtual, biopolítica del territorio y comercio internacional. Revista Fronteras, 9, 12-25.

Pengue, W. (2014). Suelos, huella de nutrientes y estabilidad ecosistémica. Revista Fronteras, 13, 1-19.

Pengue, W. (2017). El vaciamiento de las Pampas. Santiago, Chile: GEPAMA FADU UBA Fundación Heinrich Böll Cono Sur.

Pérez Manrique, P. L., Brun, J., González-Martínez, A. C., Walter, M. y Martínez Allier, J. (2013). The Biophysical Peformance of Argentina (1970 - 2009). Journal of Industrial Ecology, 17(4), 590-604.

PNUMA (2013). Tendencias del flujo de materiales y productividad de recursos en América Latina. Programa de las Naciones Unidas para el Medio Ambiente.

Prebisch, R. (1979). Biósfera y desarrollo. Seminario Regional Proyecto CEPAL/ PNUMA. Estilos de Desarrollo y Medio Ambiente en America Latina. Santiago de Chile, Chile.

Prebisch, R. (1986). El desarrollo económico de América Latina y sus principales problemas. Desarrollo Económico, 26(103).

Rimski-Korsakov, H., Rubio, G. y Lavado R. (2004) Potential nitrate losses under different agricultural practices in the pampas region, Argentina. Agricultural Water Management, 65, 83-94

Rimski-Korsakov H., Rubio, G., Pino, I. y Lavado, R. S. (2008). Destino del nitrógeno del fertilizante en un cultivo de maíz. Informaciones Agronómicas del Cono Sur, 39, 1-5.

Rockström, J., Steffen, W. L., Noone, K., Persson, Å., Chapin III, F. S., Lambin, E., ... y Nykvist, B. (2009). Planetary boundaries: exploring the safe operating space for humanity. Ecology and society, 14(2).

Schatan, J. W. (1998). El balance material de la deuda externa. Revista Ecología Política, 16, 133-139.

TEEB. (2018). TEEB for Agriculture \& Food: Scientifc and Economic Foundations. Ginebra, Suiza: UN Environment. 
Teubal, M. (2006). Expansión del modelo sojero en Argentina. De la producción de alimentos a los commodities. Realidad Económica, 220. 71-96.

Toledo, V. (2013). El metabolismo social: una nueva teoría socioecológica. Revista Relaciones, 34(136), 41-71.

UNEP (2014). Assessing Global Land Use: Balancing Consumption with Sustainable Supply. A Report of the Working Group on Land and Soils of the International Resource Panel. International Resource Panel.

Viglizzo, E. F. y Jobbagy, E. (2010). Expansión de la Frontera agropecuaria argentina y su impacto ecológico - ambiental. INTA Ediciones.

Wackernagel, M. y Rees, W. (1996). Our Ecological Footprint. Reducing Human Impact on the Earth. Six Edition. Isla Gabriola, Canadá: New Society Publishers.

Zuberman, F. (2014). Agricultura industrial y agronegocio. Una mirada desde la economía ecológica a un problema regional. En Melón, D. (Coord.), La Patria Sojera. El modelo agrosojero en el Cono Sur. Buenos Aires, Argentina: Editorial El Colectivo.

Zuberman, F. y Fernández, L. (2016). El metabolismo social en la cuenca baja del plata: Un análisis desde los outputs para evaluar las transformaciones del entorno bioproductivo. Revista Iberoamericana de Economía Ecológica, 26, 155-166. 